\title{
Primary and Secondary Prevention of Ischemic Stroke
}

\author{
Maurizio Paciaroni ${ }^{a} \quad$ Julien Bogousslavsky ${ }^{b}$ \\ a Stroke Unit and Division of Cardiovascular Medicine, University of Perugia, Perugia, Italy; ${ }^{b}$ Department of \\ Neurology and Neurorehabilitation, Swiss Medical Network, Valmont-Genolier, Glion sur Montreux, Switzerland
}

\section{Key Words}

Stroke $\cdot$ Transient ischemic attack $\cdot$ Primary prevention sures for avoiding recurrences after a first TIA or stroke manifestation, which is becoming more frequent in an increasingly ageing population.

\section{Primary Prevention of Stroke}

\section{Hypertension}

Many trials have shown the benefit of blood pressure control in all age groups and all degrees of hypertension. One study showed a 35\% reduction in total strokes and $44 \%$ reduction in fatal strokes over 5 years with a standardized blood pressure lowering regimen, compared with routine care [2]. Treatment of isolated systolic hypertension to less than $140 \mathrm{~mm} \mathrm{Hg}$ in elderly patients is also beneficial for reducing the risk of stroke [3-5]. A meta-analysis of multiple treatment trials showed that a mean reduction in diastolic blood pressure of 5-6 $\mathrm{mm} \mathrm{Hg}$ correlates with a $35-40 \%$ reduction in the incidence of stroke. This treatment effect appears valid in different races and ages. Furthermore, a meta-analysis of 9 prospective studies, which included 420,000 people followed for 10 years, found the risk continued to decrease with lower blood pressure without a lower limit in blood pressure, which was confirmed by Verdecchia et al. [6]. This suggests that antihypertensive therapy targeted toward the lowest tolerated blood pressure may be beneficial in patients at risk of stroke.
Primary prevention addresses all measures for avoiding a stroke or TIA. Secondary prevention addresses all mea-

\section{KARGER}

Fax +4161306 1234 E-Mail karger@karger.ch www.karger.com
(C) 2010 S. Karger AG, Basel 0014-3022/10/0635-0267\$26.00/0

Accessible online at:

www.karger.com/ene
Maurizio Paciaroni, MD

Stroke Unit and Division of Cardiovascular Medicine, Universiy of Perugia

Santa Maria della Misericordia Hospital, Sant'Andrea delle Fratte

IT-06126 Perugia (Italy)

Tel./Fax +39075 5782 765, E-Mail mpaciaroni@med.unipg.it 
Although lowering blood pressure is clearly beneficial, the best drug regimen to achieve this is unclear. The Antihypertensive and Lipid-Lowering Treatment to Prevent Heart Attack Trial (ALLHAT) showed that a thiazide diuretic was more effective at reducing the risk of cardiovascular events than angiotensin-converting enzyme (ACE) inhibitors or $\alpha$-blockers [7]. Patients receiving the ACE inhibitor lisinopril had a similar mortality rate to those receiving chlorthalidone, but the lisinopril group had a higher rate of stroke. Blood pressure was higher in the lisinopril group, but after adjusting for this, there was still a significant difference in stroke outcome. Issues with the restriction to monotherapy, choice of ACE inhibitor and effects among African Americans may have confounded these results. The Heart Outcomes Prevention Evaluation (HOPE) trial suggested the ACE inhibitor ramipril reduced the risk of stroke and myocardial infarction, with a 0.68 relative risk of stroke for ramipril versus placebo. Ramipril also appeared to have effects beyond that of lowering blood pressure [8]. Angiotensin II receptor blockers may also have a beneficial effect on cardiovascular events and stroke.

In the Losartan Intervention For Endpoint (LIFE) Reduction in Hypertension Study, 9,222 patients with hypertension (160-200 mm Hg/95-115 mm Hg) and signs of left ventricular hypertrophy on ECG were randomized to losartan or atenolol [9]. Patients were followed for 4 years with the goal of reducing blood pressure to $<140 / 90$ $\mathrm{mm} \mathrm{Hg}$. A thiazide diuretic was added for blood pressure control to both arms of the study. Blood pressure reduction was similar in both groups $(30.2 / 16.6 \mathrm{~mm} \mathrm{Hg}$ in the losartan group and 29.1/16.8 $\mathrm{mm} \mathrm{Hg}$ in the atenolol group). The losartan group had a $24.9 \%$ relative risk reduction of stroke over the atenolol group.

\section{Lipids}

Clinical trials analyzing the efficacy of statins have demonstrated some beneficial effects. There is evidence from randomized clinical trials that carotid plaque can regress in people with elevated cholesterol who are treated with statins. Large trials in which stroke was pre-specified as a secondary end point have shown significant reductions with statins among patients with coronary artery disease and normal or modest elevations of cholesterol $[10,11]$.

The Cholesterol and Recurrent Events (CARE) study found patients with average cholesterol levels treated with pravastatin after a myocardial infarction had a lower risk of stroke than patients receiving placebo. These patients had a serum cholesterol under $240 \mathrm{mg} / \mathrm{dl}$ with LDL cho- lesterol 115-174 mg/dl. They were followed for 5 years with outcomes of stroke or TIA. A 32\% relative risk reduction was found in patients on pravastatin versus placebo [12].

A large randomized prospective study involving over 20,000 patients followed for 5 years showed a benefit of simvastatin versus placebo in reducing mortality, stroke and myocardial infarction in high-risk vascular patients regardless of cholesterol levels. The patients studied had coronary artery disease, peripheral vascular disease or diabetes. These patients were randomly assigned to simvastatin $(40 \mathrm{mg})$ or placebo. There was a $17 \%$ reduction in vascular death, a $38 \%$ reduction in myocardial infarction and a $25 \%$ reduction in incidence of first stroke [13].

However, both the West of Scotland Coronary Prevention Study (WOSCOPS) and ALLHAT Lipid Lowering Trial did not show a reduced risk of stroke in lowerrisk patients taking a statin. ALLHAT Lipid Lowering Trial examined patients with hypertension and moderately elevated cholesterol (fasting LDL 120-189 mg/dl in patients without coronary heart disease and 100-129 $\mathrm{mg} / \mathrm{dl}$ with known coronary heart disease). Patients were randomized to treatment with $40 \mathrm{mg}$ of pravastatin or to usual care. There was no significant difference in mortality or coronary heart disease and stroke events between the two groups [14]. People with cholesterol levels above $200 \mathrm{mg} / \mathrm{dl}$ and cardiovascular risk factors should have a complete lipid analysis (total cholesterol, LDL, HDL and triglycerides) and most likely would benefit from cholesterol-lowering regimens, including statins [15]. Higher-risk patients with documented vascular disease may benefit from statins regardless of cholesterol levels.

\section{Diabetes Mellitus}

Guidelines for the management of diabetes have lowered the target fasting blood glucose level to $126 \mathrm{mg} / \mathrm{dl}$ [16]. The National Stroke Association and American Heart Association recommend rigorous comprehensive control of blood sugar levels for adherent patients with type 1 or type 2 diabetes to prevent microvascular complications [17]. Strict blood pressure control in patients with type 2 diabetes significantly lowers the risk of stroke.

\section{Cigarette Smoking}

The Nurses' Health Study and the Framingham study both demonstrated that the risk of ischemic stroke is reduced to that of nonsmokers after 2 and 5 years, respec- 
tively $[18,19]$. It has been estimated that if we could eliminate cigarette smoking in the United States, the number of strokes occurring each year would be reduced by 61,500 , and 3.08 billion stroke-related healthcare dollars would be saved [20]. The National Stroke Association and the American Heart Association recommend smoking cessation as a stroke prevention measure in accordance with guidelines issued by the Agency for Health Care Policy and Research, which address topics including screening for tobacco use, advice to quit, interventions, smoking cessation pharmacotherapy, motivation to quit and relapse prevention [21].

\section{Alcohol}

The combination of deleterious and beneficial effects of alcohol is consistent with the observation of a dosedependent relationship between alcohol and stroke. Elimination of heavy drinking can undoubtedly reduce the incidence of stroke. Because some alcohol intake, perhaps up to 2 drinks per day, may help reduce the risk of stroke, most of the public should not be discouraged from drinking in moderation [22, 23].

\section{Physical Activity}

Physical activity is of clear benefit in reducing the risk of heart disease and premature death [24]. Studies have also evaluated the association between physical activity and the risk of stroke [26-33]. The Honolulu Heart Program, which investigated older middle-aged men of Japanese ancestry, showed a protective effect of habitual physical activity from thromboembolic stroke only among nonsmokers. The Framingham study showed the benefits of combined leisure and work physical activities for men, but not for women. In the Oslo Study, among men aged $40-49$, increased leisure physical activity was related to a reduced incidence of stroke. For women aged 40-65, the Nurses' Health Study showed an inverse association between physical activity and the incidence of any stroke. In the Northern Manhattan Stroke Study, the benefits of leisure-time physical activity were noted for all ages, sexes and race-ethnic subgroups. The optimal amount of exercise needed to prevent stroke is unclear, particularly for the elderly. Among subjects in a case-control study in West Birmingham (UK) who were free of cardiovascular disease, recent vigorous exercise was no more protective than walking. Among the older cohort of the Framingham study, the strongest protection was detected in the medium tertile physical activity subgroup, with no benefit from additional activity. The protective effect of physical activity may be partly mediated through its role in controlling risk factors such as hypertension, diabetes and obesity. Biological mechanisms such as increased HDL and reduced homocysteine levels may also be responsible for the effect of physical activity $[34,35]$.

\section{Dietary Factors and Obesity}

Dietary intake of fruits and vegetables may reduce the risk of stroke. These foods may protect against stroke through antioxidant mechanisms or by raising potassium levels [36-39]. Dietary antioxidants, including vitamin C, vitamin E and beta-carotene, belong to a group of antioxidants called flavonoids, which are found in fruits and vegetables. These scavengers of free radicals are thought to be associated with stroke risk reduction through the free-radical oxidation of LDL, which inhibits the formation of atherosclerotic plaques [40]. The large Western Electric cohort found a moderate decrease in stroke risk associated with a higher intake of both betacarotene and vitamin C [41]. Other dietary factors associated with a reduced risk of stroke include milk, calcium [42] and fish oils [43, 44]. Clinical trials using vitamin E, beta-carotene and vitamin $\mathrm{C}$ have not demonstrated convincing results for reducing the risk of stroke.

\section{Hyperhomocysteinemia}

Another important dietary component is homocysteine. Epidemiological studies have demonstrated an association between moderately elevated homocysteine and vascular disease, including stroke [45]. Genetic and environmental causes of increased serum homocysteine have been implicated as a modifiable determinant of cardiovascular and cerebrovascular events [46, 47]. The Framingham study found that deficiencies in folate, B12 and pyridoxine accounted for the majority of elevated homocysteine levels in the study cohort. Additionally, evidence from case-control studies has suggested that increased dietary and supplemental intake of vitamin B6 may decrease stroke risk [48]. However, the large prospective $\mathrm{Vi}$ tamin in Stroke Protection (VISP) trial did not show a benefit with treatment with B6, B12 or folate in preventing recurrent stroke [49].

\section{Estrogen Use}

There have been conflicting reports on hormone replacement therapy and the risk of stroke. The most recent studies have shown that postmenopausal hormone replacement therapy does not lower the risk of cardiovascular events or stroke. In the Heart and Estrogen/Progestin Replacement Studies (HERS I and II), patients on hormone replacement therapy had an equivalent risk of 
cardiac or stroke events as patients on placebo [50]. The Women's Health Initiative study on hormone replacement therapy and stroke showed an increased risk of stroke in women taking estrogen [51]. More than 16,000 women were followed for a mean of 5.6 years. Some $1.8 \%$ of the estrogen/progestin group had a stroke, compared with $1.3 \%$ of the placebo group. Some $79.8 \%$ of these strokes were ischemic. The hazard ratio for women on hormones versus placebo was 1.31 . Hormone replacement therapy in postmenopausal women should not be used for primary prevention of stroke and, in fact, may increase the risk of ischemic stroke.

\section{Asymptomatic Carotid Disease}

The role of prophylactic endarterectomy in people with asymptomatic extracranial carotid artery stenosis is controversial. A few randomized controlled trials have addressed this issue. The largest was the Asymptomatic Carotid Atherosclerosis Study (ACAS) [52]. Patients ( $\mathrm{n}=$ 1,662 ) were randomized to surgery plus best medical therapy or to best medical therapy without carotid endarterectomy. Angiographic complications occurred in $1.2 \%$ and the perioperative stroke risk was $2.3 \%$. After a median follow-up of 2.7 years, the study was stopped early because a significant benefit of surgery was found. The rate of ipsilateral stroke, any perioperative stroke or death in surgically treated patients was estimated at $5 \%$ over 5 years, while in medically treated patients the rate was $11 \%$ ( $55 \%$ risk reduction, $\mathrm{p}=0.004$ ). The benefit was most notable among men, and there was no relationship between benefit and the degree of carotid artery stenosis. It was also clear from the absolute risks that any perioperative complication rates greater than the $2.3 \%$ for stroke or death reported in the ACAS trial would eliminate the potential benefit of the operation. Another trial, the Asymptomatic Carotid Surgery Trial (ACST), concluded that in subjects younger than 75 years with severe carotid stenosis, carotid endarterectomy approximately halves the net 5 -year risk of stroke. Stroke risk at 5 years in the immediate surgery group was $3.8 \%$, but $11 \%$ in the deferred surgery group. The immediate, 30 -day risk of stroke or death after endarterectomy was 3.1\%.This does not mean that all patients should undergo surgery, but that careful selection is required and the risk of surgery has to be below $3 \%$ to be beneficial [53].

\section{Atrial Fibrillation}

Numerous large clinical trials have demonstrated the efficacy of warfarin for preventing stroke among patients with nonvalvular atrial fibrillation (AF). The relative risk reduction ranged from 42 to $86 \%$ for warfarin versus placebo. Warfarin was also found to be safe: the annual rate of major bleeding was $1.3 \%$ on warfarin and $1 \%$ on placebo or aspirin. The benefit of anticoagulation clearly outweighs the risks: for 1,000 patients with nonvalvular AF treated with warfarin for 1 year, 35 thromboembolic events can be prevented at a cost of one major bleed. The role of aspirin for the prevention of stroke in patients with AF is controversial, with only the Stroke Prevention in Atrial Fibrillation (SPAF I) trial showing a modest risk reduction for $325 \mathrm{mg}$ of aspirin versus placebo. However, in the SPAF III trial, warfarin at an INR of 2-3 was found to be superior to a mini-dose of warfarin and $325 \mathrm{mg}$ of aspirin. However, the disadvantages of warfarin treatment, including the need for careful monitoring and dose adjustment, together with the risk of serious bleeding, have led to the quest for an alternative approach to prevention of ischemic stroke in patients with AF. An antithrombotic agent that might be used (once it has been approved) instead of warfarin is the oral direct thrombin inhibitor, dabigatran. In patients with AF, dabigatran given at a dose of $110 \mathrm{mg}$ was associated with rates of stroke and systemic embolism that were similar to those associated with warfarin, as well as lower rates of major hemorrhage. Dabigatran administered at a dose of $150 \mathrm{mg}$, as compared with warfarin, was associated with lower rates of stroke and systemic embolism, but similar rates of major hemorrhage [54].

In patients with $\mathrm{AF}$ for whom vitamin $\mathrm{K}$ antagonist therapy was unsuitable, the addition of clopidogrel to aspirin reduced the risk of major vascular events (relative risk with clopidogrel 0.89 ; 95\% CI $0.81-0.98, \mathrm{p}=0.01$ ), especially stroke, and increased the risk of major hemorrhage (relative risk 1.57; 95\% CI 1.29-1.92, p < 0.001) [55].

\section{Secondary Prevention}

After a patient has had a stroke or TIA, there are several strategies for preventing a recurrence. These depend on the mechanism of the stroke or TIA. Controlling risk factors is very important and the studies discussed for primary prevention also apply to recurrent stroke prevention, although the number of trials available supporting this hypothesis are fewer than for primary prevention.

\section{Hypertension}

Few studies have demonstrated significant benefits with hypotensive drugs in secondary prevention of stroke 
with different results, mainly related to the different hypotensive drugs used.

The HOPE study investigated the effect of ramipril in patients who were at high risk for cardiovascular events. Since $11 \%$ of included patients had a prior stroke, an approach to stroke secondary prevention efficacy could be made in this subgroup of patients, showing a nonsignificant $17 \%$ reduction in the relative risk of stroke recurrence [56].

PROGRESS [57] was a randomized trial of a perindopril-based blood pressure lowering regimen among 6,105 individuals with a previous stroke or TIA. Patients were randomly assigned active treatment $(n=3,051)$ or placebo $(n=3,054)$. The study was designed to determine the effects of a blood pressure lowering regimen in hypertensive and nonhypertensive patients with a history of stroke or TIA. Active treatment consisted of a flexible regimen based on the perindopril ( $4 \mathrm{mg}$ daily), with the addition of the diuretic indapamide at the discretion of treating physician. The primary outcome was total stroke (fatal or nonfatal). Over 4 years of follow-up, active treatment reduced blood pressure by $9 / 4 \mathrm{~mm} \mathrm{Hg} ; 307$ (10\%) in the active group and $420(14 \%)$ in the placebo group (relative risk reduction $28 \%, 95 \%$ CI 17-38). Active treatment also reduced the risk of total major vascular events (relative risk reduction 26\%, 95\% CI 16-34). There were similar reductions in the risk of stroke in hypertensive and nonhypertensive subgroups. Combination therapy with perindopril plus indapamide reduced blood pressure by $12 / 5$ $\mathrm{mm} \mathrm{Hg}$ and stroke risk by $43 \%$. Single drug therapy reduced blood pressure by $5 / 3 \mathrm{~mm} \mathrm{Hg}$ and produced no discernable reduction in the risk of stroke. In PROGRESS, the analysis of the specific ischemic stroke subtypes demonstrated a significant benefit of active treatment with the combination of perindopril and indapamide in reducing atherothrombotic (relative risk reduction 39\%) and lacunar strokes (relative risk reduction 23\%), as well as hemorrhagic stroke (relative risk reduction 50\%), with no effects on cardioembolic strokes. The HYVET study provided evidence that antihypertensive treatment with indapamide, with or without perindopril, is also beneficial in persons 80 years or older [58].

In the MOSES trial, a total of 1,405 well-defined, highrisk hypertensives with a cerebral event during the last 24 months were randomized to eprosartan (an angiotensin II type receptor antagonist) or nitrendipine (calcium antagonist; mean follow-up 2.5 years) [59]. The primary end point was the composite of total mortality and all cardiovascular and cerebrovascular events, including all recurrent events. Blood pressure was reduced to a comparable extent without any significant differences between the two groups during the whole study period $(150.7 / 84 \mathrm{~mm}$ $\mathrm{Hg}$ and $152.0 / 87.2 \mathrm{~mm} \mathrm{Hg}$ with eprosartan and to $137.5 / 80.8 \mathrm{~mm} / \mathrm{Hg}$ and $136.0 / 80.2 \mathrm{~mm} / \mathrm{Hg}$ with nitrendipine therapy, respectively, confirmed by ambulatory blood pressure monitoring). During follow-up, a total of 461 primary events occurred: 206 with eprosartan and 255 with nitrendipine (incidence density ratio 0.79; 95\% CI 0.66-0.96). There were 77 eprosartan and 101 nitrendipine cardiovascular events (incidence density ratio 0.75; 95\% CI 0.55-1.02) and 102 eprosartan and 134 nitrendipine cerebrovascular events (incidence density ratio 0.75 ; 95\% CI 0.58-0.97).

A meta-analysis of the trials described below, excluding the MOSES trial, reported that antihypertensive therapy was effective in reducing recurrent stroke (OR 0.76), myocardial infarction (OR 0.79) and vascular events (OR $0.79)$ [60]. Heterogeneity between drug classes was apparent: $\beta$-receptor antagonists did not seem to reduce any vascular events, diuretics alone reduced stroke but not myocardial infarction, and angiotensin-converting inhibitors reduced myocardial infarction but not stroke. However, the most effective intervention was dual therapy (angiotensin-converting inhibitors and diuretics), which reduced each of the three outcomes. This systematic review did not include angiotensin II receptor blockers since no data of secondary prevention randomized clinical trials were available. Another more recent systematic review confirmed the importance of blood pressure reduction in primary and secondary stroke prevention, and in a meta-regression analysis, calcium channel blockers were found to be better than angiotensin-converting inhibitors in stroke prevention [61]. The conclusion after this meta-analysis was that lowering blood pressure is an effective method for reducing the risk of subsequent stroke. Most patients will need $\geq 2$ drugs, and combinations should be logical and based on class pharmacological activities (especially taking into account effects on the renin system) [62].

In a combined analysis of PROFESS and TRASCEND, the incidence of the composite of stroke, myocardial infarction or vascular death was $12.8 \%$ for telmisartan versus $13.8 \%$ for placebo (hazard ratio 0.91 ; $95 \%$ CI $0.85-$ $0.98, \mathrm{p}=0.013)[63]$

\section{Estrogen Use}

Primary prevention studies did not show that hormone replacement therapy was beneficial for lowering the risk of stroke in postmenopausal women. It has also not been shown to lower risk of recurrent stroke in wom- 
en who have had a stroke or TIA [64]. In one study, there was also a nonsignificant trend towards worse outcome after stroke in women who were taking estrogen.

\section{Lipids}

Lipid-lowering agents have mostly been tested in patients with coronary artery disease and recent myocardial infarction, and results from subpopulations with stroke or TIA are most relevant in terms of primary prevention. However, more recent data indicate that statins may be effective in reducing stroke recurrence. Effects are mostly not due to lipid lowering, but rather reflect 'pleiotropic effects', which are still not completely understood. A subgroup analysis of the Heart Protection Study showed a relative risk reduction of $4.9 \%$ in patients with a qualifying cerebrovascular event treated with $40 \mathrm{mg}$ of simvastatin, irrespective of their initial lipid values [13]. Due to the small number of stroke patients included in this big trial $(1,820$ out of a total of 20,536$)$, this result did not reach statistical significance. While PROSPER failed to show similar effects (for various reasons, still being discussed avidly by experts) [65], the first analysis of the Anglo-Scandinavian Cardiac Outcome Trial Lipid Lowering Trial (ASCOTLLT) showed - for the first time - a significant reduction in nonfatal ischemic strokes in patients with a first TIA or stroke who were treated with atorvastatin $(10 \mathrm{mg} /$ day $)$ versus placebo. There is still some concern about extrapolating these results to stroke patients because most studies to date have included patients without a history of stroke and who were younger than the typical stroke population. Furthermore, the sex ratio (8:2 male:female in myocardial infarction trials) differed from that usually seen in stroke trials (5:4 male:female), and the rate of hypertension (the main risk factor for stroke patients) was rather low in patients with myocardial infarction (26\% in the $4 \mathrm{~S}$ trial, $40 \%$ in LIPID and CARE, but $55-60 \%$ in stroke trials). This is reflected by low general event rates of stroke (3-4\%) compared with that in typical stroke trials $(6-8 \%)$.

The Stroke Prevention by Aggressive Reduction of Cholesterol Levels (SPARCL) study was the first to investigate the effect of statins on the risk for cerebrovascular events in patients without a history of coronary artery disease. This double-blind, randomized, placebo-controlled, multicenter trial examined the effect of aggressive atorvastatin therapy $(80 \mathrm{mg} /$ day $)$ on specified cerebrovascular end points. Patients were eligible for the study if they had had a previous transient ischemic attack or stroke, had an LDL level between $100 \mathrm{mg} / \mathrm{dl}(2.58$ $\mathrm{mmol} / \mathrm{l})$ and $190 \mathrm{mg} / \mathrm{dl}(4.91 \mathrm{mmol} / \mathrm{l})$, and had no evi- dence of coronary artery disease. The primary clinical end point was the time to first occurrence of a fatal or nonfatal stroke. In this study, 4,731 patients who had had a stroke or TIA within the past 6 months were randomized. After 6 years of follow-up, 265 patients in the atorvastatin group had had a fatal or nonfatal stroke compared with 311 in the control group. There was a 16\% risk reduction in time to first occurrence of stroke with atorvastatin (adjusted HR 0.84, 95\% CI 0.71-0.99; NNT = 46). For the secondary end point of time to stroke or TIA, there was a $23 \%$ risk reduction $(\mathrm{HR}=0.77,95 \%$ CI 0.67 0.88 ) with 375 events in the atorvastatin group and 476 in controls. Moreover, there was a $35 \%$ reduction of coronary events (HR 0.65, 95\% CI 0.49-0.87) [66].

Theoretically, lipid-lowering medications may increase the risk of cerebral hemorrhage. In a meta-analysis including 83,205 subjects participating in previous trials, an increase in the rate of intracerebral hemorrhage among patients treated with statins was not observed when used for primary prevention (OR 0.90, 95\% CI 0.76-1.05), but an increased incidence of hemorrhagic stroke was observed in secondary prevention trials (OR 1.7, 95\% CI 1.19-2.50) [68]. In the SPARCL study, of the 88 patients who had at least one intracerebral hemorrhage, 55 were in the atorvastatin group and 33 were in the placebo group. In this trial, the relative risk of intracerebral hemorrhage increased by $66 \%$ among patients in the atorvastatin group. The overall benefit in terms of stroke risk reduction was significant despite the increase in intracerebral hemorrhage in the atorvastatin group. Secondary analyses of the SPARCL study have been conducted to address the implications of statin therapy for patients having intracerebral hemorrhages during the trial. A preliminary exploratory analysis suggests that brain hemorrhage is not related to a major lowering of $\mathrm{LDL}$ cholesterol levels; however power may be lacking here, thereby not showing a lower threshold effect. To date, study results suggest that statins have a good overall safety profile, but despite this, further studies need to be carried out to exclude that statin use in secondary prevention of stroke does not cause brain hemorrhage.

\section{Carotid Endarterectomy}

Carotid endarterectomy has been shown to reduce the risk of ischemic stroke in patients with symptomatic carotid stenosis. The North American Symptomatic Carotid Endarterectomy Trial (NASCET) studied patients with TIA or minor stroke and an ipsilateral carotid stenosis of $70 \%$ or more. This study was stopped early because of the significant benefit seen in the surgical group. It found the 
2-year risk of ipsilateral stroke was $9 \%$ in the surgical group and 26\% in the medical group (aspirin 1,300 mg/ day). The absolute risk reduction was $17 \%$. Risk reductions were less for those with moderate stenosis (50-69\%) and dependent on concomitant risk factors [68]. The Veterans Administration Cooperative Study showed that among those with carotid stenosis $>50 \%$, the risk of stroke after a mean follow-up of 11.9 months was $7.7 \%$ in the surgical group and $19.4 \%$ in the nonsurgical group. The European Carotid Surgery Trial (ECST) also showed a benefit for high-grade symptomatic carotid stenosis, but there was no significant benefit of surgery for those with $0-29 \%$ stenosis [69]. The consensus is that for patients with a TIA or minor stroke and ipsilateral carotid stenosis of more than $70 \%$, carotid endarterectomy is the best option for preventing a recurrent event. For those with $<50 \%$ stenosis, endarterectomy has no benefits, while for those with $50-69 \%$ stenosis and ipsilateral symptoms, the use of endarterectomy depends on the risk strata of the patient.

\section{Angioplasty and Stent}

Nonsurgical treatment of carotid disease with angioplasty and stent placement through endovascular techniques is becoming more widespread. It is now a technically feasible option and has been used in patients who are not good surgical candidates, either because of the location of the stenosis or because of a high risk for undergoing anesthesia. Studies comparing angioplasty with carotid endarterectomy have addressed issues of safety and long-term recurrent stroke risk. In a small study, 43 patients with symptomatic carotid stenosis $(\geq 70 \%)$ underwent stent placement. Of these, 40 patients had successful recanalization. They were followed for a mean of 20 months. Mortality at 30 days was $2.5 \%$ and the overall stroke or death rate was $5 \%$ at the end of the follow-up period [70]. The Wallstent study was stopped early because of worse outcome in patients who underwent stent placement over endarterectomy. The 30 -day risk of stroke or death was 11 versus 5\%. The Carotid and Vertebral Artery Transluminal Angioplasty Study (CAVATAS) was a larger, randomized study comparing angioplasty and endarterectomy [71]. Some 504 patients with symptomatic carotid stenosis deemed necessary for treatment and amenable to either procedure were randomized. The study found no significant difference in death or stroke between the two groups (10\%). There was no difference in death, disabling stroke or nondisabling stroke. There was a lower incidence of cranial nerve palsy in the interventionally treated patients (0 vs. $9 \%$ ). The Stenting and
Angioplasty with Protection in Patients at High Risk of Endarterectomy (SAPPHIRE) study randomized 307 high-risk patients to stenting or endarterectomy. These patients had a history of concurrent cardiac disease. Some $70 \%$ of the patients had asymptomatic carotid stenosis. Patients in the angioplasty group had a better 30 -day outcome, with stroke or death occurring in $4.5 \%$ versus $6.6 \%$ in the endarterectomy group. The 30-day outcome of stroke, death or myocardial infarction was $5.8 \%$ in the angioplasty group versus $12.6 \%$ in the surgical group [72].

The International Carotid Stenting Study (ICSS) [73], the largest trial of endarterectomy versus endovascular treatment, reported a nearly 2 -fold higher procedural risk of stroke in the endovascular treatment group. Even without the inclusion of the results of ICSS and the other trials that reported only the periprocedural risks, a meta-analysis of all the available data on long-term outcome in randomized trials of endovascular treatment versus endarterectomy for symptomatic carotid stenosis showed a significantly worse outcome after endovascular treatment [74].

\section{Antiplatelets}

The antiplatelet drugs aspirin, ticlopidine, clopidogrel and dipyridamole have been shown to be efficacious in the secondary prevention of stroke.

\section{Aspirin}

Numerous clinical trials have compared aspirin with placebo for the prevention of stroke and death after TIA or minor stroke. The Antithrombotic Trialists' Collaboration used meta-analysis to combine the results. In their analyses of 21 trials of people with a past history of stroke or TIA, they reported an odds reduction of $22 \%$ for nonfatal stroke, nonfatal myocardial infarction or vascular death with a 2 -year risk of $17.8 \%$ for those treated with antiplatelets and $21.4 \%$ for controls [75]. Similar reductions were found for women and men, young and old, hypertensives and normotensives, and diabetics and nondiabetics. Recommendations for aspirin dosage range from $30 \mathrm{mg}$ per day to $1,300 \mathrm{mg}$ per day. In the Antithrombotic Trialists' Collaboration meta-analysis, aspirin $\geq 75 \mathrm{mg}$ was equivalent to $<75 \mathrm{mg}$ in preventing vascular events. The risk of extracranial bleeding was similar with aspirin doses $<325 \mathrm{mg}$. The OR was 1.7 for aspirin $<325 \mathrm{mg}$ compared with controls. The OR was 1.5 for doses $<75 \mathrm{mg}$ and 1.4 for doses of $160-325 \mathrm{mg}$.

A new meta-analysis of the Antithrombotic Trialists' Collaboration has confirmed that in the secondary prevention trials, aspirin allocation yields a greater absolute reduction in serious vascular events (6.7 vs. $8.2 \%$ per year, 
$\mathrm{p}<0.0001$ ), with a nonsignificant increase in hemorrhagic stroke, but reductions of about a fifth in both total stroke ( 2.08 vs. $2.54 \%$ per year, $\mathrm{p}=0.002)$ and coronary events ( 4.3 vs. $5.3 \%$ per year, $\mathrm{p}<0.0001$ ) [76].

Current recommendations are to treat stroke and TIA survivors with 50-325 mg.

\section{Ticlopidine}

Another antiplatelet agent that has proven efficacy in stroke prevention is ticlopidine. In the Canadian American Ticlopidine Study, ticlopidine was compared with placebo in a triple-blind, randomized, multicenter study after a completed noncardioembolic stroke among 1,053 patients. Ticlopidine resulted in a $23 \%$ risk reduction in an intention-to-treat analysis and a $30 \%$ reduction in the efficacy analysis. The risk reduction for nonfatal or fatal recurrent stroke was 33\%. In the Ticlopidine Aspirin Stroke Study, ticlopidine was compared with aspirin $(1,300 \mathrm{mg} /$ day $)$ among 3,069 patients with TIA or minor stroke. Overall, there was a $12 \%$ reduction in stroke or death at 3 years, but a $47 \%$ risk reduction in fatal or nonfatal stroke was observed during the first year for those treated with ticlopidine compared with aspirin. Benefits were found among both men and women and the effect was observed in the subgroup with minor stroke. The African-American Antiplatelet Stroke Study, however, failed to show any benefit of ticlopidine compared with aspirin in this high-risk population [77]. The use of ticlopidine is now rarely recommended for the prevention of stroke recurrence, and the drug was discontinued in the UK in February 2003.

\section{Dipyridamole}

Older studies indicated that dipyridamole had a similar benefit to aspirin for prevention of recurrent stroke. Results from the European Stroke Prevention Study have indicated that $200 \mathrm{mg}$ b.i.d. of extended-release dipyridamole was as effective as $25 \mathrm{mg}$ b.i.d. of aspirin, with the combination of the two being even better than aspirin alone for the prevention of stroke after TIA or minor stroke. The relative risk reduction of stroke or death was $13 \%$ for aspirin, $15 \%$ for dipyridamole and $24 \%$ for the combination of the two [78].

The ESPRIT trial found that primary outcome events (composite of death from all vascular causes, nonfatal stroke, nonfatal myocardial infarction or major bleeding complication) arose in $13 \%$ patients on aspirin and dipyridamole and in $16 \%$ on aspirin alone (hazard ratio 0.80 , 95\% CI $0.66-0.98$; absolute risk reduction $1.0 \%$ per year, 95\% CI 0.1-1.8) [79].

\section{Clopidogrel}

The antiplatelet agent clopidogrel was studied in the Clopidogrel versus Aspirin in Patients at Risk of Ischemic Events (CAPRIE) trial. In this study, 19,185 patients with ischemic stroke, myocardial infarction or peripheral vascular disease were randomized to $75 \mathrm{mg}$ of clopidogrel versus $325 \mathrm{mg}$ of aspirin. For the primary end point of combined stroke, myocardial infarction or vascular death, there was a relative risk reduction of $8.7 \%$ and an absolute risk reduction of $0.9 \%$ with clopidogrel over aspirin. Among the stroke subgroup, there was a 7.3\% relative risk reduction of stroke, myocardial infarction or vascular death [80].

\section{Combination Regimens}

Combination antiplatelet regimens such as extendedrelease dipyridamole plus aspirin have proven efficacy for prevention of stroke or death after stroke or TIA. Other antiplatelet combinations are under investigation, including clopidogrel plus aspirin. The Management of Atherothrombosis with Clopidogrel in High-Risk Patients with Recent TIA or Stroke (MATCH) trial randomized high-risk patients (prior ischemic stroke, myocardial infarction, peripheral vascular disease or diabetes) to therapy with clopidogrel or clopidogrel plus aspirin [81]. The authors assessed for outcomes of ischemic stroke, myocardial infarction or death at 18 months. There was no significant difference in outcome for the two treatment groups, but the combination of clopidogrel and aspirin significantly increased the risk of major bleeding events.

The PROFESS trial did not meet the predefined criteria for noninferiority, but showed similar rates of recurrent stroke with aspirin associated with dipyridamole and with clopidogrel [82]. There is no evidence that either of the two treatments was superior to the other in the prevention of recurrent stroke.

\section{Warfarin}

Warfarin is an oral anticoagulant that has been demonstrated to be effective in the prevention of cardioembolic stroke. Randomized clinical trials have evaluated the relative merits of warfarin or aspirin in patients with nonvalvular AF. The European Atrial Fibrillation Trial convincingly demonstrated that anticoagulation therapy reduced the risk of recurrent stroke in patients with $\mathrm{AF}$ and TIA or minor stroke from 12 to $4 \%$, compared with placebo. The risk reduction of $67 \%$ was similar to that found in the other AF trials among people with no prior neurological events. Oral anticoagulants were more ef- 
fective than aspirin, and aspirin was better than placebo, but the latter effect was not significant. This clinical trial, in conjunction with the other warfarin studies, provides support that warfarin is the therapy of choice in patients with a cardiac source and a TIA or minor stroke, provided there is no contraindication to its use. There has been considerable debate about the use of warfarin after noncardioembolic stroke or TIA. The Warfarin Aspirin Recurrent Stroke Study (WARSS) was designed to answer the question of whether warfarin had a role in the treatment of these strokes [83]. WARSS was a randomized, double-blind trial of warfarin with an INR of 1.4-2.8 versus aspirin $(325 \mathrm{mg})$ in 2,206 patients with noncardioembolic stroke. These patients were followed for 2 years with the primary end point of stroke or death. Death or recurrent stroke occurred in $16.9 \%$ of patients. There was no difference between patients treated with warfarin or aspirin. There was also no difference in hemorrhage rates. Although warfarin with an INR of 1.4-2.8 appears to be safe, there was no increased benefit in preventing recurrent stroke compared with aspirin. In contrast, another recently published study investigated warfarin and aspirin in symptomatic intracranial arterial stenosis. In this trial warfarin was associated with significantly higher rates of adverse events and provided no benefit over aspirin. The authors recommended the use of aspirin in preference to warfarin for patients with intracranial arterial stenosis [84]. A more recent (although not randomized), long-term, follow-up trial with careful warfarin monitoring suggested quite a remarkable reduction of stroke recurrences without more adverse events. Antiplatelets are the treatment of choice for prevention of stroke after noncardioembolic stroke or TIA.

\section{Other Measures}

There are virtually no data on the significance of smoking cessation, abstinence from alcohol or the treatment of diabetes for secondary prevention of stroke or TIA, although the merit of these measures seems to be self-evident. The effects on stroke recurrence of obesity and lack of physical exercise have not been investigated. A recent study suggests that folic acid and vitamin B6and B12-supplements lower the risk of recurrent stroke, but the results are not statistically significant [85].

\section{Patent Foramen Ovale and Other Cardioembolic Sources}

A patent foramen ovale (PFO) is an embryological remnant that may persist in some people. It is a communication between the two atria, which allows blood to be shunted from the right to the left atrium. This may cause paradoxical embolism to the brain. Two case-control studies using contrast transthoracic echocardiography among young patients with ischemic stroke found a significant association between $\mathrm{PFO}$ and stroke. Other casecontrol and cross-sectional studies found PFO to be a stroke risk factor even in older patients. The risk persisted after controlling for other factors such as hypertension and diabetes mellitus. Because the prevalence of PFO in the general population may be greater than $20 \%$, it could account for a large number of strokes. It is clear that most people with a PFO do not develop a stroke. Therefore, other factors are likely to mediate this risk, for example: size of the PFO, presence of a venous thrombosis or coexistence of a hypercoagulable state.

Although PFO may increase the risk of a first stroke, it does not appear to increase the risk of recurrent stroke. For example, the presence and size of a PFO did not have a significant effect on stroke recurrence rate in the PFO in Cryptogenic Stroke Study (PICSS) [86], and patients treated with warfarin did not have a significant difference in stroke rate compared with patients treated with aspirin. Other studies investigating the efficacy of PFO closure devices are underway.

Another recently recognized potential cardiac stroke risk factor is the presence of an atrial septal aneurysm. This is a congenital malformation of the atrial septum causing bulging of the septum into either the left or the right atrium. Case-control series have found an increased frequency of atrial septal aneurysms among patients with unexplained stroke, compared with control patients referred for an echocardiogram for other clinical reasons. Some studies have found the presence of atrial septal aneurysm to increase the risk of stroke among young patients with PFO. However, PICSS did not detect a difference in recurrent stroke rate in patients with $\mathrm{PFO}$ and atrial septal aneurysm, compared with those without. There was also no difference in treatment effects of warfarin or aspirin.

Aortic arch atheromas may also be a risk factor for stroke. Transesophageal echocardiography can classify the presence, thickness, character (presence of ulceration or mobile components) and location of any aortic atheroma. In the largest autopsy series to date, Amarenco and Labreuche [67] detected a significantly greater frequency of ulcerated aortic atheromas in patients who had died from a stroke than in patients who had died from other neurological diseases. The greatest frequency of ulcerated atheromas was found in patients with cryptogenic stroke. Using transesophageal echo- 
cardiography, the same authors found a $14.4 \%$ frequency of atheromas with a thickness $\geq 4 \mathrm{~mm}$ in patients older than 60 , compared with a $2.2 \%$ frequency in controls. Other studies with transesophageal echocardiography have also shown a high frequency of protruding aortic atheromas in patients with cryptogenic stroke, and have confirmed the role of aortic atheromas as an independent risk factor for stroke. It is still not clear whether aortic atheromas are the cause of the stroke or a marker of more diffuse and severe atherosclerotic disease. Currently, there are no clinical trial data to support anticoagulation for any of these potential causes of cardioembolic stroke.

\section{Conclusions}

Stroke prevention is a crucial issue, given that stroke is a frequent and severe disorder, and acute stroke therapies, which are effective at the individual level, have only a limited impact on public health. Vascular risk factors should be treated to prevent ischemic stroke, especially high blood pressure, high blood cholesterol and cigarette smoking. To reduce the risk of any new vascular events after a first ischemic stroke or TIA, complementary strategies are an optimal management of stroke risk factors for all types of strokes and TIA, with antithrombotic therapy and carotid surgery in severe symptomatic stenosis.

\section{References}

1 Lloyd-Jones D, Adams R, Carnethon M, et al: American Heart Association: Heart disease and stroke. Statistics 2009. Update. Circulation 2009;119:e1-e161.

-2 Five-year findings of the hypertension detection and follow-up program. III. Reduction in stroke incidence among persons with high blood pressure. Hypertension Detection and Follow-up Program Cooperative Group. JAMA 1982;247:633-638.

3 Prevention of stroke by antihypertensive drug treatment in older persons with isolated systolic hypertension. Final results of the Systolic Hypertension in the Elderly Program (SHEP). SHEP Cooperative Research Group. JAMA 1991;265:3255-3264.

4 Party MW: Medical Research Council trial of treatment of hypertension in older adults: principal results. BMJ 1992;304:405-412.

5 Dahlöf B, Lindholm LH, Hansson L, et al: Morbidity and mortality in the Swedish Trial in Old Patients with Hypertension (STOPHypertension). Lancet 1991;338:1281-1285.

-6 Verdecchia P, Staessen JA, Angeli F, et al: Usual versus tight control of systolic blood pressure in non-diabetic patients with hypertension (Cardio-Sis): an open-label randomised trial. Lancet 2009;374:525-533.

7 ALLHAT Officers and Coordinators for the ALLHAT Collaborative Research Group. The Antihypertensive and Lipid-Lowering Treatment to Prevent Heart Attack Trial: Major outcomes in high-risk hypertensive patients randomized to angiotensin-converting enzyme inhibitor or calcium channel blocker vs. diuretic: The Antihypertensive and Lipid-Lowering Treatment to Prevent Heart Attack Trial (ALLHAT). JAMA 2002; 288:2981-2997.

$\checkmark 8$ HOPE Investigators: Effects of an angiotensin-converting enzyme inhibitor, ramipril, on cardiovascular events in high-risk patients. N Engl J Med 2000;342:145-153.
9 Dahlöf B, Devereux R, Kjeldsen S, et al: Cardiovascular morbidity and mortality in the Losartan Intervention for Endpoint reduction in hypertension study (LIFE): a randomized trial against atenolol. Lancet 2002; 359:995-1003.

10 Sacks F, Pfeffer M, Moye L, et al: The effects of pravastatin on coronary events after myocardial infarction in patients with average cholesterol levels. N Engl J Med 1996;335: 1001-1009.

11 Long-Term Intervention with Pravastatin in Ischaemic Disease (LIPID) Study Group: Prevention of cardiovascular events and death with pravastatin in patients with coronary heart disease and a broad range of initial cholesterol levels. N Engl J Med 1998;339: 1349-1357.

12 Plehn J, Davis B, Sacks F, et al: Reduction of stroke incidence after myocardial infarction with pravastatin: the Cholesterol and Recurrent Events (CARE) study. The CARE Investigators. Circulation 1999;99:216-223.

$13 \mathrm{MRC} / \mathrm{BHF}$ Heart protection study of cholesterol lowering with simvastatin in 20536 high-risk individuals: a randomized placebo-controlled trial. Lancet 2002;360:7-22.

14 ALLHAT Collaborative Research Group: Major outcomes in moderately hypercholesterolemic, hypertensive patients randomized to pravastatin vs. usual care. JAMA 2002;288:2998-3007.

15 Summary of the National Cholesterol Education Program (NCEP) Adult Treatment Panel II Report. JAMA 1993;269:3015-3023.

16 Moghissi ES, Korytkowski MT, DiNardo $\mathrm{MM}$, et al: American Association of Clinical Endocrinologists and American Diabetes Association consensus statement on inpatients glycemic control. Diabetes Care 2009; 32:1119-1131.
17 Gorelick P, Sacco R, Smith D, et al: Prevention of a first stroke: a review of guidelines and a multidisciplinary consensus statement from the National Stroke Association. JAMA 1999;281:1112-1120.

18 Kamachi I, Colditz G, Stampfer M, et al: Smoking cessation and decreased risk of stroke in women. JAMA 1993;269:232-236.

19 Wolf P, D’Agostino R, Kannel W, et al: Cigarette smoking as a risk factor for stroke: the Framingham Study. JAMA 1988;259:10251029.

20 Gorelick P: Stroke prevention: windows of opportunity and failed expectations - a discussion of modifiable cardiovascular risk factors and a prevention proposal. Neuroepidemiology 1997;16:163-173.

21 US Preventive Services Task Force: Guide to Clinical Prevention Services: Report to the US Preventive Services Task Force, vol XCII. Baltimore, Williams \& Wilkins, 1996.

22 Mukamal KJ, Ascherio A, Mittleman MA, Conigrave KM, Camargo CA Jr, Kawachi I, Stampfer MJ, Willett WC, Rimm EB: Alcohol and risk for ischemic stroke in men: the role of drinking patterns and usual beverage. Ann Intern Med 2005;142:11-19.

23 Bos S, Grobbee DE, Boer JM, Verschuren WM, Beulens JW: Alcohol consumption and risk of cardiovascular disease among hypertensive women. Eur J Cardiovasc Prev Rehabil 2010;17:119-126.

24 NIH Consensus Development Panel: Physical activity and cardiovascular health. JAMA 1996;276:241-246.

25 Abbott R, Rodriguez B, Burchfiel C, Curb J: Physical activity in older middle-aged men and reduced risk of stroke: the Honolulu Heart Program. Am J Epidemiol 1994;139: 881-893.

26 Fletcher G: Exercise in the prevention of stroke. Health Reports 1994;6:106-110. 
27 Kiely D, Wolf P, Cupples L, et al: Physical activity and stroke risk: the Framingham Study. Am J Epidemiol 1994;140:608-620.

28 Haheim L, Holme I, Hjermann I, Leren P: Risk factors of stroke incidence and mortality. A 12-year follow-up of the Oslo Study. Stroke 1993;24:1484-1489.

-29 Gillum R, Mussolino M, Ingram D: Physical activity and stroke incidence in women and men - the NHANES I Epidemiologic Followup Study. Am J Epidemiol 1996;143:860-869.

- 30 Sacco R, Gan R, Boden-Albala B, et al: Leisure-time physical activity and ischaemic stroke risk: the Northern Manhattan Stroke Study. Stroke 1998;29:380-387.

- 31 Wannamethee G, Shaper A: Physical activity and stroke in British middle aged men. BMJ 1992;304:597-601.

32 Manson J, Stampfer M, Willett W, et al: Physical activity and incidence of coronary heart disease and stroke in women. Circulation 1995;91(Suppl):5.

33 Lee I, Hennekens C, Berger K, et al: Exercise and risk of stroke in male physicians. Stroke 1999;30:1-6

- 34 Williams P: High-density lipoprotein cholesterol and other risk factors for coronary heart disease in female runners. $\mathrm{N}$ Engl J Med 1996;334:1298-1303.

- 35 Nygard O, Vollset S, et al: Total plasma homocysteine and cardiovascular risk profile - the Hordaland Homocysteine Study. JAMA 1995;274:1526-1533.

36 Gillman M, Cupples L, Posner B, et al: Protective effects of fruits and vegetables on development of stroke in men. JAMA 1995;273: 1113-1117.

- 37 Gey K, Stahelin H, Eichholzer M: Poor plasma status of carotene and vitamin $\mathrm{C}$ is associated with higher mortality from ischaemic heart disease and stroke. Clin Invest Med 1993;71:3-6.

38 Khaw K, Barrett-Connor E: Dietary potassium and stroke-associated mortality. N Engl J Med 1987;316:235-240.

- 39 Benson R, Jacobs B, Boden-Albala B, et al: Vitamin E intake: a primary preventive measure in stroke. Neurology 1999;52:A146.

-40 Diaz M, Frei B, Vita J, Keaney J: Antioxidants and atherosclerotic heart disease. N Engl J Med 1997;282:408-416.

-41 Daviglus ML, Orencia AJ, Dyer AR, et al: Dietary vitamin $C$, beta-carotene and 30-year risk of stroke: results from the Western Electric Study. Neuroepidemiology 1997;16:6977.

42 Abbott R, Curb D, Rodriguez B, et al: Effect of dietary calcium and milk consumption on risk of thromboembolic stroke in older middle-aged men. The Honolulu Heart Study. Stroke 1996;27:813-818

43 Orenica A, Daviglus M, Dyer A, et al: Fish consumption and stroke in men. 30-year findings of the Chicago Western Electric Study. Stroke 1996;27:204-209.
44 Morris M, Manson J, Rosner B, et al: Fish consumption and cardiovascular disease in the Physicians' Health Study: a prospective study. Am J Epidemiology 1995;142:166-175.

45 Ueland P, Refsum H, Brattstrom L: Plasma homocysteine and cardiovascular disease; in: Francis RB (ed): Atherosclerotic Cardiovascular Disease, Hemostatis, and Endothelial Function. New York, Marcel Dekker, 1992, pp 183-236.

46 Giles W, Croft J, Greenlund K, et al: Total homocyst(e)ine concentration and the likelihood of nonfatal stroke - results from the Third National Health and Nutrition Examination Survey, 1988-1994. Stroke 1998;29: 2473-2477.

47 Sacco R, Roberts J, Jacobs B: Homocysteine as a risk factor for ischaemic stroke: an epidemiological story in evolution. Neuroepidemiology 1998; 17:167-173.

48 Jacobs B, Sacco R, Lui R, et al: Low dietary intake of vitamin $\mathrm{B} 6$ is associated with an increased risk of ischaemic stroke. Stroke 1999; 30:252.

49 Toole JF, Malinow MR, Chambless LE, et al: Lowering homocysteine in patients with ischemic stroke to prevent recurrent stroke, myocardial infarction, and death: the Vitamin Intervention for Stroke Prevention (VISP) randomized controlled trial. JAMA 2004;291:565-575.

50 Grady D, Herrington D, Bittner V, et al: Cardiovascular disease outcomes during 6.8 years of hormone therapy: Heart and Estrogen/Progestin Replacement Study follow-up (HERS II). JAMA 2002;288:49-57.

51 Wassertheil-Smoller S, Hendrix S, Limacher $\mathrm{M}$, et al: Effect of estrogen plus progestin on stroke in postmenopausal women. The Women's Health Initiative: a randomized trial. JAMA 2003;289:2673-2684.

52 Executive Committee for the Asymptomatic Carotid Atherosclerosis Study: Endarterectomy for asymptomatic carotid atherosclerosis. JAMA 1995;273:1421-1428.

53 MRC Asymptomatic Carotid Surgery Trial (ACST) Collaborative Group: Prevention of disabling and fatal strokes by successful carotid endarterectomy in patients without recent neurological symptoms: randomised controlled trial. Lancet 2004;363:1491-1502.

54 Connolly SJ, Ezekowitz MD, Yusuf S, et al: Dabigatran versus warfarin in patients with atrial fibrillation. N Engl J Med 2009;361: 1139-1151.

55 Connolly SJ, Pogue J, Hart RG, et al: Effect of clopidogrel added to aspirin in patients with atrial fibrillation. N Engl J Med 2009;360: 2066-2078.

- 56 Yusuf S, Sleight P, Pogue J, Bosch J, Davies R, Dagenais G: Effects of an angiotensin-converting-enzyme inhibitor, ramipril, on cardiovascular events in high-risk patients. The Heart Outcomes Prevention Evaluation Study Investigators. N Engl J Med 2000;342: 145-153.
57 PROGRESS Collaborative Group: Randomized trial of a perindopril-based blood-pressure-lowering regimen among 6,105 individuals with previous stroke or transient ischaemic attack. Lancet 2001;358:10331042.

58 Beckett NS, Peters R, Fletcher AE, Staessen JA, et al: Treatment of hypertension in patients 80 years of age or older. N Engl J Med 2008;358:1887-1898.

59 Schrader J, Luders S, Kulschewski A, Hammersen F, Plate K, Berger J, et al: Mortality and morbidity after stroke - eprosartan compared with nitrendipine for secondary prevention: principal results of a prospective randomized controlled study (MOSES). Stroke 2005;36:1218-1226.

60 Rashid P, Leonardi-Bee J, Bath P: Blood pressure reduction and secondary prevention of stroke and other vascular events: a systematic review. Stroke 2003;34:2741-2748.

61 Zhang H, Thijs L, Staessen JA: Blood pressure lowering for primary and secondary prevention of stroke. Hypertension 2006;48: 187-195.

62 Bath PM, Sprigg N: Control of blood pressure after stroke. Hypertension 2006;48. 203-204.

63 Diener HC: Preventing stroke: the PROFESS, ONTARGET, and TRASCEND trial programs. J Hypertens 2009;27(Suppl 5):S31S36.

64 Viscoli C, Brass L, Kernan W, et al: A clinical trial of estrogen-replacement therapy after ischaemic stroke. N Engl J Med 2001;345: 1243-1249.

65 Fiorenza AM, Sommariva D, Branchi A: The PROSPER trial. Lancet 2003;361:428.

66 SPARCL Investigators: High-dose atorvastatin after stroke or transient ischemic attack. N Engl J Med 2006;355:549-559.

67 Amarenco P, Labreuche J: Lipid management in the prevention of stroke: review and updated meta-analysis of statins for stroke prevention. Lancet Neurol 2009;8:453-463.

68 Sacco R: Extracranial carotid stenosis. N Engl J Med 2001;345:1113-1118.

69 ECST Investigators: Interim results for symptomatic patients with severe (70-99\%) or with mild $(0-29 \%)$ carotid stenosis. Lancet 1991;337:1235-1243.

70 Dietz A, Kerkefeld J, Theron J, et al: Endovascular treatment of symptomatic carotid stenosis using stent placement: long-term follow-up of patients with a balanced surgical risk/benefit ratio. Stroke 2001;32:1855-1859.

71 CAVATAS Investigators: Endovascular versus surgical treatment in patients with carotid stenosis in the Carotid and Vertebral Artery Transluminal Angioplasty Study (CAVATAS): a randomized trial. Lancet 2001;357:1729-1737.

72 Gurm HS, Yadav JS, Fayad P, et al: Long-term results of carotid stenting versus endarterectomy in high risk patients: the Sapphire Study. N Engl J Med 2008;358:1572-1579. 
73 Brown MM, Ederle J, Bonati LH, et al: Safety results of the International Carotid Stenting Study. Early outcome of patients randomised between carotid stenting and endarterectomy for symptomatic carotid stenosis. European Stroke Conference Stockholm, May 2009.

74 Rothwell PM: Poor outcome after endovascular treatment of symptomatic carotid stenosis: time for a moratorium. Lancet Neurology 2009;8:871-873.

-75 Antithrombotic Trialists' Collaboration: Collaborative meta-analysis of randomised trials of antiplatelet therapy for prevention of death, myocardial infarction, and stroke in high risk patients. BMJ 2002;324:71-86.

-76 Antithrombotic Trialists' Collaboration: Aspirin in the primary and secondary prevention of vascular disease: a collaborative meta-analysis of individual participant data from randomised trials. Lancet 2009;373: 1849-1860.
77 Gorelick P, Richardson D, Kelly M, et al: Aspirin and ticlopidine for prevention of recurrent stroke in black patients: a randomized trial. JAMA 2003;289:2947-2957.

78 Diener H, Cunha L, Forbes C, et al: European Stroke Prevention Study. 2. Dipyridamole and acetylsalicylic acid in the secondary prevention of stroke. J Neurol Sci 1996;143:1-13.

79 Halkes PH, van Gijn J, Kappelle LJ, et al: Aspirin plus dipyridamole versus aspirin alone after cerebral ischemia of arterial origin (ESPRIT): randomised controlled trial. Lancet 2006;367:1665-1673.

80 CAPRIE Committee: A randomized, blinded, trial of clopidogrel versus aspirin in patients at risk of ischaemic events (CAPRIE). Lancet 1996;348:1329-1339.

81 Diener HC, Bogousslavsky J, Brass LM et al, MATCH investigators. Aspirin and clopidogrel compared with clopidogrel alone after recent ischaemic stroke or transient ischaemic attack in high-risk patients $(\mathrm{MATCH})$ randomised, double-blind, placebo-controlled trial. Lancet 2004;364:331-337.
82 Sacco RL, Diener HC, Yusuf S, et al: Aspirin and extended-release dipyridamole versus clopidogrel for recurrent stroke. N Engl J Med 2008;359:1238-1251.

83 Mohr J, Thompson J, Lazar R, et al: A comparison of warfarin and aspirin for the prevention of recurrent ischaemic stroke. N Engl J Med 2001;345:1444-1451.

84 Chimowitz MI, Lynn, MJ, Howlett-Smith H, et al: Comparison of warfarin and aspirin for symptomatic intracranial arterial stenosis. N Engl J Med 2005;352:1305-1316.

-85 Toole JF, Malinow MR, Chambless LE, et al: Lowering homocysteine in patients with ischemic stroke to prevent recurrent stroke, myocardial infarction, and death: the Vitamin Intervention for Stroke Prevention (VISP) randomized controlled trial. JAMA 2004;291:565-575.

86 Homma S, Sacco R, Di Tullio M, et al: Effect of medical treatment in stroke patients with patent foramen ovale: patent foramen ovale in Cryptogenic Stroke Study. Circulation 2002;105:2625-2631. 